\title{
Recording 4D-STEM Datasets at a Range of Beam Tilts Simultaneously with Multi- Beam Electron Diffraction
}

Colin Ophus ${ }^{1}$, Xuhao Hong ${ }^{2}$, Steven Zeltmann ${ }^{3}$, Karen Bustillo ${ }^{4}$, Rohan Dhall ${ }^{5}$, Benjamin Savitzky ${ }^{4}$, Alexander Mueller ${ }^{4}$ and Andrew Minor ${ }^{1,3}$

${ }^{1}$ Lawrence Berkeley National Lab, Berkeley, California, United States, ${ }^{2}$ Nanjing University, Nanjing, Jiangsu, China (People's Republic), ${ }^{3}$ University of California - Berkeley, Berkeley, California, United States, ${ }^{4}$ National Center for Electron Microscopy (NCEM), Molecular Foundry, Lawrence Berkeley National Laboratory, Berkeley, California, United States, ${ }^{5}$ Molecular Foundry, Lawrence Berkeley National Lab, Berkeley, California, United States,

Transmission electron microscopy (TEM) is an enormously versatile tool for imaging, diffraction, and spectroscopy in materials science and biology. In one TEM experimental geometry, nanobeam electron diffraction experiment (NBED), a moderately-converged electron probe is scanned across a sample in a $2 \mathrm{D}$ grid of probe positions, and a probe diffraction image is recorded at each position. Modern high-speed electron detectors allow the recording of many thousands or even millions of diffraction patterns from a given sample, over length scales ranging from nanometers to micrometers [1]. These diffraction patterns can be used to solve crystal structures, map a sample's structure and orientation, or directly measure sample structure with differential phase contrast (DPC) or ptychographic imaging [1]. However, due to the large curvature of the Ewald sphere given by the high accelerating voltages of a TEM instrument, a crystalline sample might only fall into the Bragg condition within a very small angular range for a given crystallographic zone axis. When a crystalline sample in an NBED experiment does not produce a 2D pattern of diffracted Bragg disks, the crystallography of the sample cannot be uniquely determined [2]. This means that in polycrystalline samples, many crystallites can be missed in an NBED experiment.

One obvious solution to this problem is to record NBED datasets at various tilt angles, filling in the sphere of possible Bragg diffraction conditions. This however increases the experimental time required, and beam-sensitive samples might be damaged or changed during tilt experiments. Another solution introduced in [3] is called precession electron diffraction (PED), where the electron beam is rotated continuously around a cone of tilt angles during data collection. This will excite a wider range of Bragg conditions and thus produce more complete diffraction images. Additionally, because PED averages many diffraction conditions, it can reduce multiple scattering effects and give more kinematical estimates of the crystal diffraction intensities [4]. However, PED requires careful alignment of the beam tilt and probe descan in order to produce sharp diffraction patterns. Additionally, it is not possible to separate out the different beam tilt angles in a typical PED experiment.

In this talk we introduce an alternative method of simultaneously recording diffraction patterns with multiple beam tilts: multi-beam electron diffraction (MBED). We produce these beams with a modified condenser system in a ThermoFisher Titan instrument similarly to [5], with a schematic and some experimental results shown in Figure 1. In this experiment, eight simultaneous diffraction patterns were recorded from a single crystal of gold, covering an angular range of +/- 3 degrees. Each diffraction pattern contains a unique cut through the reciprocal lattice from the same parent crystal, demonstrating that these beams are intersecting the sample at different angles. The coherence of this experiment was specifically set so that each beam was self-coherent, but with no mutual coherence between beams in order to facilitate 
simple interpretation of the diffraction patterns. Additionally, each beam-forming aperture has a unique shape, allowing the diffraction patterns to be separated from each other even while overlapping.

In this talk, we will present experimental and simulated MBED results. We will discuss several potential MBED applications: (1) mapping sample structure, orientation and strain, (2) solving crystal structures of large single crystals, (3) "precession without precessing," i.e. gaining the benefits of PED without requiring beam precession, (4) multiple simultaneous DPC experiments measuring the sample electron field at different orientations, and (5) tomographic measurements in real and reciprocal space that do not require sample or beam tilting. We will show that MBED is particularly well-suited to in situ measurements, where the sample structure might change too quickly to allow for tilting [6].

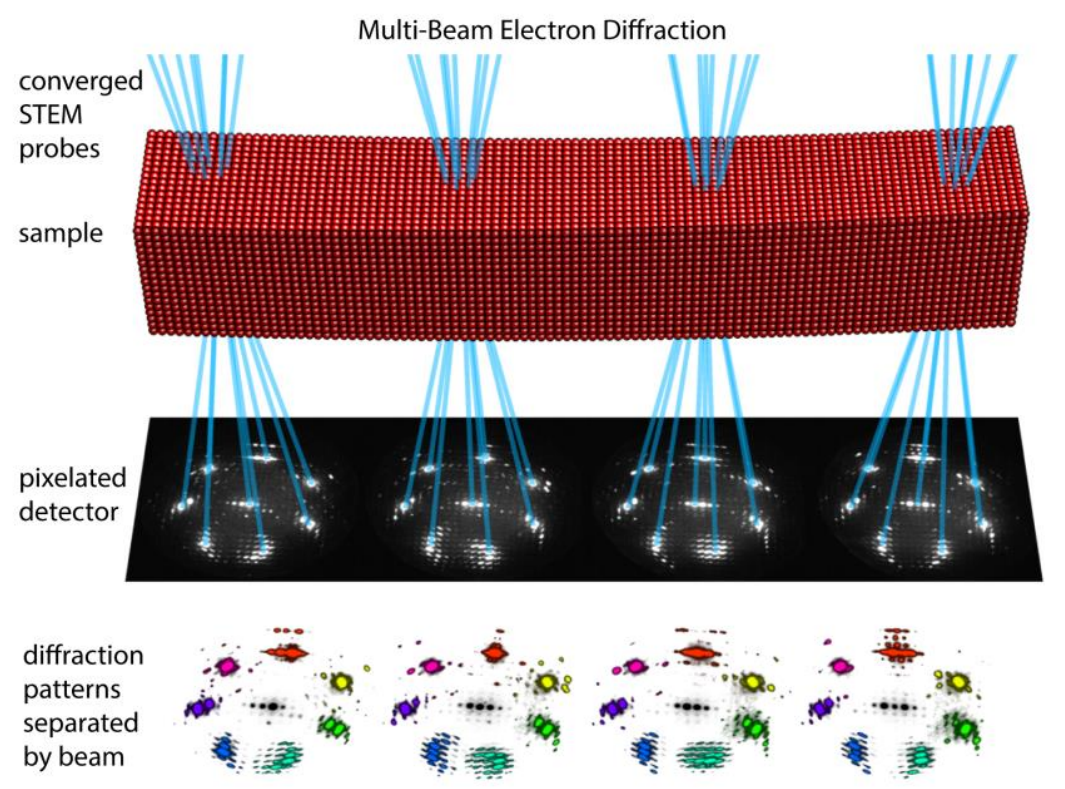

Figure 1. Experimental implementation of multi-beam electron diffraction. Multiple probe positions including a central beam along the optical axis and seven beams equally spaced around a cone tilted 3 degrees from the optical axis. These diffraction patterns were recorded from a gold sample on a pixelated detector. Below, the eight unique diffraction patterns have been separated into different colors, and tilted to emphasize that they are different slices through diffraction space.

\section{References}

[1] C Ophus, Microscopy and Microanalysis 25, 563 (2019).

[2] Williams and Carter, Transmission electron microscopy: Diffraction, Imaging, and Spectrometry, Springer (2016).

[3] R Vincent and P Midgley, Ultramicroscopy 53, 271 (1994).

[4] P Midgley and AS Eggeman, IUCrJ, 2, 126 (2015).

[5] SE Zeltmann et al., Ultramicroscopy 209, 112890 (2020).

[6] Work at the Molecular Foundry was supported by the Office of Science, Office of Basic Energy Sciences, of the U.S. Department of Energy under Contract No. DE-AC02-05CH11231. CO acknowledges additional support from the U.S. Department of Energy Early Career Research Award Program. SEZ was supported by STROBE, a NSF Science and Technology Center. BHS acknowledges support from the Toyota Research Institute. 